\title{
Potentiation of responding on a VR schedule by a stimulus correlated with reinforcement: Effects of diffuse and localized signals
}

\author{
PHIL REED, TODD R. SCHACHTMAN, and GEOFFREY HALL \\ University of York, York, England
}

\begin{abstract}
In Experiment 1, rats were trained to leverpress on a variable ratio (VR) 30 schedule with a 500-msec delay between the reinforced response and food delivery. Subjects that experienced a signal during the delay responded faster than did control subjects that received the stimulus uncorrelated with reinforcement. Higher response rates were obtained when the stimulus used to signal reinforcement was auditory rather than visual. Experiments 2 and 3 compared the effects of signaling reinforcement with either a localized or a diffuse light on responding maintained by VR schedules of reinforcement. Elevated response rates were observed with the diffuse stimulus, but the localized stimulus failed to produce such potentiation. Experiment 3 also examined the conditioned reinforcing power of localized and diffuse visual stimuli. These results are discussed with reference to (1) theories of selective association and sign tracking and (2) their implications for current theories of signaling reinforcement.
\end{abstract}

For rats responding on a variable interval (VI) schedule of reinforcement, the presentation of a stimulus in a brief delay between the response that satisfies the schedule and food delivery results in a lower response rate than is seen when such a stimulus is absent or is presented uncorrelated with reinforcement (Pearce \& Hall, 1978; St. Claire-Smith, 1979; Tarpy, Lea, \& Midgley, 1983; Tarpy, Roberts, Lea, \& Midgley, 1984). Several explanations have been offered for this effect. It has been suggested that when the stimulus signals reinforcement it will overshadow the response and restrict the growth of a response-reinforcer association (Pearce \& Hall, 1978; St. Claire-Smith, 1979); that the signal comes to evoke patterns of behavior that compete with the measured response of leverpressing (Iversen, 1981); and that the signal enhances the animals' sensitivity to the time-based characteristics of the VI schedule and, thus, promotes more efficient (i.e., less frequent) responding (Roberts, Tarpy, \& Lea, 1984; but see also Tarpy, St. Claire-Smith, \& Roberts, 1986).

The impetus for this last account came from the observation that presenting a signal along with the reinforcer had no effect on response rate when behavior was supported by a variable ratio (VR) schedule (Tarpy et al., 1983; but see also Dickinson, Peters, \& Shecter, 1984). Tarpy et al. (1983) argued that the efficiency theory predicted no effect, because, with a VR schedule, the availability of the reinforcement is not determined by tem-

\footnotetext{
This research was supported by a grant from the United Kingdom Science and Engineering Research Council to G. Hall. We thank J. E. Wright, University of Leeds, for his support and the loan of equipment, and $\mathrm{C}$. Bonardi for her comments on an earlier version of this manuscript. Requests for reprints may be addressed to any of the authors, Department of Psychology, University of York, Heslington, York Y01 5DD, England.
}

poral factors as it is on a VI schedule. Doubt is cast upon this interpretation, however, by the results of a recent study by Reed, Schachtman, and Hall (in press). It was found that signaling reinforcement on a VR schedule actually produced an enhanced rate of response, compared with that shown by subjects given uncorrelated presentations of the stimulus (see also Morgan, Lea, \& Nicholas, 1976, Experiment 4). This result, which Reed et al. (in press) referred to as a potentiation effect, is not to be expected on the basis of any of the theories currently available, which were devised to deal with the overshadowing effect seen on VI schedules. As a first step toward devising some new account that can accommodate all of the phenomena, it seems important to confirm the reliability of the potentiation effect and to determine the source of the discrepancy between the results of Reed et al. (in press) and those of Tarpy et al. (1983).

Reed et al. (in press) pointed out two procedural differences between their study and that of Tarpy et al. (1983). The latter used a smaller ratio schedule (i.e., VR-10 as opposed to VR-30) and gave less extensive training. Reed et al. (in press) presented evidence to suggest that the magnitude of the potentiation effect is reduced with these parameters. A third difference concerns the nature of the signal: Reed et al. (in press) used an auditory stimulus (a 500-msec tone), whereas Tarpy et al. (1983) used a visual stimulus (the illumination of a jewel lamp for $400 \mathrm{msec}$ ). This procedural difference assumes importance in light of the possible role played by sign tracking in these experiments. Rats tend to approach and make contact with the source of a discrete stimulus correlated with reinforcement (Iversen, 1981; Roberts, Tarpy, \& Cooney, 1985). Such autoshaped responding can interfere with leverpressing (Karpicke, Christoph, Petersen, \& Hearst, 1977) and therefore tend to reduce the observed rate of 
response. A diffuse auditory stimulus will of course also support conditioned responding (e.g., Holland, 1977), but the responses produced seem less likely to interfere with leverpressing (see Edgar, Hall, \& Pearce, 1981). The effectiveness of the signal in potentiating responding may then be revealed only when a nonlocalized stimulus is employed. The present experiments were designed to evaluate this suggestion and its implications.

\section{EXPERIMENT 1}

In this experiment we attempted to replicate the potentiation effect of Reed et al. (in press) and to determine whether the use of an auditory cue is critical in producing the effect. To this end, we compared the effects of signaling reinforcement with an auditory stimulus, previously used by Reed et al. (in press), with the effects produced with a visual stimulus (the illumination of a jewel light), as used by Tarpy et al. (1983).

\section{Method}

Subjects. Thirty-two naive male Lister hooded rats served as subjects. The rats were 4-6 months old at the start of the study, and were experimentally naive. The subjects' ad-lib weights ranged from 275 to $360 \mathrm{~g}$, and they were maintained at $80 \%$ of these weights. The animals were housed in pairs with water constantly available in the home cage.

Apparatus. Four identical operant chambers (Campden Instruments Ltd.) were employed. Each chamber was housed in a lightand sound-attenuating case. A background masking noise (65 dBA re SPL) was supplied by a ventilating fan. Each chamber was equipped with two retractable levers, one of which was withdrawn for the duration of the study. The food tray was centrally located between the two levers. Reinforcement consisted of one $45-\mathrm{mg}$ food pellet. Located above the lever was a small jewel light fixnure $(3 \mathrm{~W})$ that could be operated from a 24-V power supply to provide the visual stimulus. The auditory stimulus was a $100-\mathrm{dBA}$ tone ( $25 \mathrm{dBA}$ above background) supplied by a speaker mounted on top of the chamber. No houselight was employed in the study.

Procedure. Animals were first given two 30-min sessions of magazine training, during which the levers were retracted from the chamber and food pellets were delivered on a fixed-time 60 -sec schedule. During the first session of magazine training, the flap covering the food tray was raised to allow easy access to the pellets. For the second session the flap was lowered to its standard position. Following magazine training, the left lever was inserted into the box and remained in place for the rest of the study. Subjects were then trained to leverpress on a continuous reinforcement (CRF) schedule. Two sessions of CRF were given, each being terminated after 75 reinforcements had been earned.

All subsequent sessions consisted of 30 trials, that is, $\mathbf{3 0}$ reinforcements were earned before the subject was removed from the chamber. The subjects were exposed to one session of a VR-5 schedule, one session of VR-10, and two sessions of VR-20. Following this pretraining, subjects were divided into four groups $(n=8)$ that were matched for response rate on the last session of VR-20. Two of these groups were to receive the light as a stimulus and two the tone. Of the two groups in each stimulus modality condition, one received a brief signaled delay of reinforcement $(500 \mathrm{msec})$ following the criterion response of a VR-30 schedule (the range for the VR-30 schedule was 5-55). The other group received the same delay of reinforcement but with a 500-msec response-contingent signal presented randomly with respect to reinforcement. Phase 1 lasted for $\mathbf{4 0}$ sessions and then the signaling contingencies were reversed (although the animals still experienced the same stimulus modality as in Phase 1, i.e., the light or the tone). The reversal of the contingencies in Phase 2 lasted for 24 sessions.

\section{Results and Discussion}

On the last day of pretraining, the mean response rates for the to-be-signaled and to-be-unsignaled groups for the light condition were 27.82 and 26.55 responses per minute, respectively. Equivalent scores for the tone condition were 27.07 and 27.00 responses per minute. The response rates for both phases of Experiment 1 are displayed as four-session blocks in Figure 1.

Inspection of the results for Phase 1 shows that when reinforcement was signaled by the tone, response rates were rapidly elevated compared with those shown by the uncorrelated-tone control group. However, the difference in response rate between the two groups receiving the light as a stimulus took longer to emerge, and this difference never matched that generated between the tone groups. The uncorrelated-light control condition produced the same relatively low response rate as did the uncorrelatedtone condition.

A three-factor analysis of variance (ANOVA) with reinforcement signal, stimulus modality, and blocks as factors was carried out on the response rates over the 10 foursession blocks of Phase 1. This ANOVA revealed statistically significant main effects of signal $[F(1,28)=5.74$, $p<.001]$ and block $[F(9,252)=71.71, p<.001]$. However, the main effect of modality was not significant $[F(1,28)=1.97, p>.10]$. Of the interactions, only that of signal and block was statistically significant $[F(4,253)$ $=4.91, p<.0011$.

When the signaling contingencies for the tone animals were reversed in Phase 2, the signal-induced potentiation of responding readily reversed. The tone as a signal for reinforcement supported higher response rates than did the uncorrelated tone. The response rates in the final block were comparable to the levels of responding for the corresponding signal conditions in Phase 1. In contrast, the reversal of the signaling contingencies for the light groups abolished the difference that had emerged at the end of Phase 1, but failed to reestablish the enhanced response rates in the signaled condition. A three-factor ANOVA (signal $\times$ modality $\times$ block) conducted on the response rates for all groups over Phase 2 revealed no significant main effect of signal $[F(1,28)=2.94, p>.05]$ or of modality $[F(1,28)=2.83, p>.05]$, but a significant main effect of block $[F(5,140)=19.80, p<.05]$. The interactions between signal and modality and between signal and block proved to be nonsignificant ( $p s>10$ ). The interaction between modality and block, however, was statistically significant $[F(5,140)=3.55, p<.05]$, as was the three-way interaction $[F(5,140)=3.47, p<$ .011 .

Despite the fact that there was no statistically significant effect of stimulus modality on the production of potentiation, it is clear from an examination of Phase 1 that a large numerical difference exists between the two 


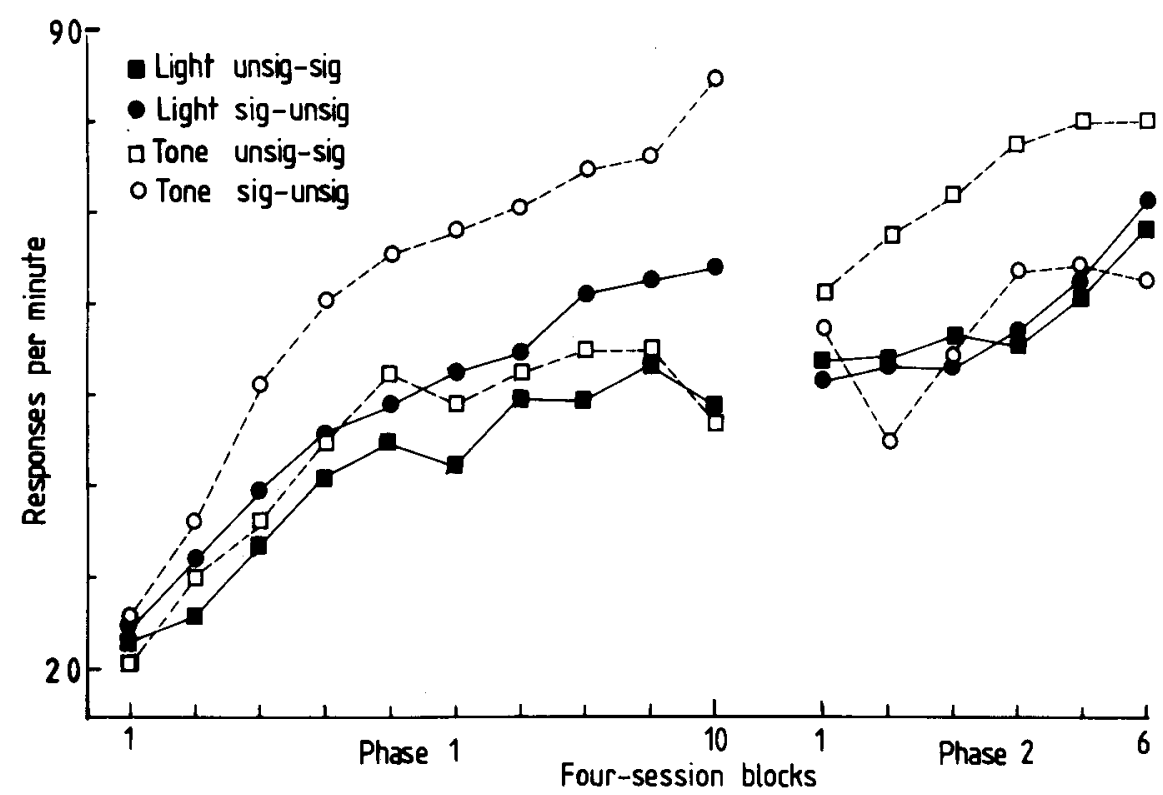

Figure 1. Results from Experiment 1. Mean response rates represented as four-session blocks for all four groups: sig = signaled reinforcement; unsig = stimulus presented uncorrelated with reinforcement.

signaled groups (light vs. tone). Further support for the differential effectiveness of the two stimuli in enhancing response rates is given by the lack of a reversal in response rates when the signaling contingencies were reversed for the light groups. In contrast, rates produced by reversing the signaling contingencies for the tone groups rapidly reversed. To confirm the effectiveness of the tone, but not the light, in producing a potentiation effect, response rates generated on the terminal block of each phase were analyzed. With signaling contingency as a within-subject factor, a three-factor ANOVA (signal $\times$ modality $\times$ phase) was conducted on the response rates generated on the last blocks of each phase. This analysis revealed no main effect of modality $(F<1)$. There were statistically significant main effects of signal $[F(1,14)=78.14, p<$ $.001]$ and phase $[F(1,14)=13.81, p<.01]$, and significant interactions of signal and modality $[F(1,14)=$ $28.00, p<.001]$ and signal and phase $[F(1,14)=8.34$, $p<.05]$. No other interaction proved to be significant $(p s>.10)$. The interaction was further examined by separate two-factor ANOVAs (signal $\times$ phase) conducted on the tone and light groups. Analysis of response rates for the tone groups revealed a main effect of signal $[F(1,7)$ $=153.47, p<.001]$, but no main effect of phase and no interaction $(F \mathrm{~s}<1)$. In contrast, neither main effect nor the interaction was significant for the light groups $(p>.20)$. These analyses corroborate the suggestion that the light was not as effective as the tone in enhancing responding. This experiment, therefore, successfully replicated the potentiation effect of Reed et al. (in press). It also showed that using a visual signal like that used by Tarpy et al. (1983) can attenuate (Phase 1) or abolish (Phase 2) the potentiation effect.

\section{EXPERIMENT 2}

The results of Experiment 1 support the suggestion that previous failures to find a potentiation effect with a VR schedule stemmed in part from the use of a visual stimulus as a signal. They are also consistent with the suggestion that the tone produces potentiation and the light does not because the latter stimulus comes to evoke competing patterns of behavior. However, alternative interpretations are possible. In particular, although the light and tone used in Experiment 1 did not appear to differ in salience, it may be that such stimuli differ intrinsically in the readiness with which they form associations with a food reinforcer. For pigeons, visual stimuli acquire greater control over responding than do auditory stimuli when the reinforcer is food, even when the auditory cue is one that in other circumstances is perfectly effective (Foree \& LoLordo, 1976). In rats, the situation may be reversed (see Harsh, Badia, \& Ryan, 1984). If, in Experiment 1 , the tone was more readily associated with food than was the light, then the potentiation effect (which may depend upon the development of such an association) should emerge more readily in the subjects given the tone as a signal for food.

In Experiment 2, we attempted to distinguish between the account outlined above and that based upon the notion of sign tracking. We compared the effects of a diffuse visual signal associated with reinforcement with those of the discrete localized light used in Experiment 1 . If visual signals form the relevant association only slowly, then any potentiation effect should develop at the same (relatively slow) rate in both cases. However, these two stimuli should differ in the readiness with which they tend to sup- 
port autoshaped competing behavior, with the diffuse stimulus producing less such behavior than the localized stimulus (Hearst \& Jenkins, 1974; Holland, 1977). The account that attributes the attenuation of potentiation with the light in Experiment 1 to autoshaped competing responses would lead to the expectation that potentiation might emerge readily with the diffuse light.

\section{Method}

Subjects and Apparatus. The 16 subjects that formed the two light groups in Experiment 1 served again in this study. The apparatus was as described in Experiment 1, with the following modifications. A Perspex ceiling was fitted in the chamber to allow a diffuse illumination of the chamber from a $30-\mathrm{W}$ strip light mounted above the ceiling.

Procedure. The animals responded on a VR-60 schedule, but experienced the same signaling contingencies as they had in Phase 2 of Experiment 1. In Phase 1 of the present study the animals either had reinforcement signaled by the localized light or received presentations of the localized stimulus uncorrelated with reinforcement. This phase lasted for eight sessions, each session consisting of 30 trials. Following this, the animals remained on the VR- 60 schedule and experienced the same signaling contingencies as in Phase 1, except that the diffuse overhead light was used instead of the localized jewel light. This phase lasted for 24 sessions, each consisting of 30 trials. Following this treatment, the subjects experienced 10 more sessions of the same schedule and signaling conditions as described above, except that the localized visual stimulus was again used as the stimulus.

\section{Results and Discussion}

Group mean response rates, represented as two-session blocks, over the three phases of the study are displayed in Figure 2. Inspection of the response rates for Phase 1 suggests that the subject that received uncorrelated presentations of the localized stimulus tended to respond slightly faster than did the subject that received signaled reinforce- ment. This difference was not, however, statistically reliable. A two-factor ANOVA (signal $\times$ block) was conducted on the response rates for Phase 1 . This analysis revealed no statistically significant main effect of signal $[F(1,14)=3.07, p>.10]$ and no interaction of signal $\times$ block $(F<1)$. However, there was a statistically significant main effect of block $[F(3,42)=6.75, p<.001]$. At the introduction of the diffuse stimulus, the numerical difference between the groups was first abolished and subsequently reversed, as the group that had reinforcement signaled eventually came to display higher response rates than did the group receiving uncorrelated presentations of the diffuse stimuli. At the reintroduction of the localized visual stimulus, this difference in response rates was abolished.

Statistical analysis confirmed the above description of the results. A two-factor ANOVA (signal $\times$ block) conducted on the response rates of Phase 2 demonstrated no statistically significant main effect of signal $(F<1)$, but a main effect of block $[F(11,154)=3.73, p<.001]$ and a statistically significant interaction of these two factors $[F(11,154)=2.09, p<.05]$. A further analysis of the terminal performance achieved in this phase demonstrated that the diffuse signal came to support a higher response rate over the last three two-session blocks. A two-factor ANOVA (signal $\times$ block) revealed a statistically significant main effect of signal $[F(1,14)=5.89, p<.05]$, but no main effect of block or interaction between these two factors $(p s>.20)$. Analysis of the response rates in Phase 3 revealed no main effect of signal or block, nor was the interaction of these two factors significant $(p>.10)$.

The results of the present experiment show that elevated response rates can be obtained by signaling reinforcement with a visual stimulus when that stimulus is diffuse, but

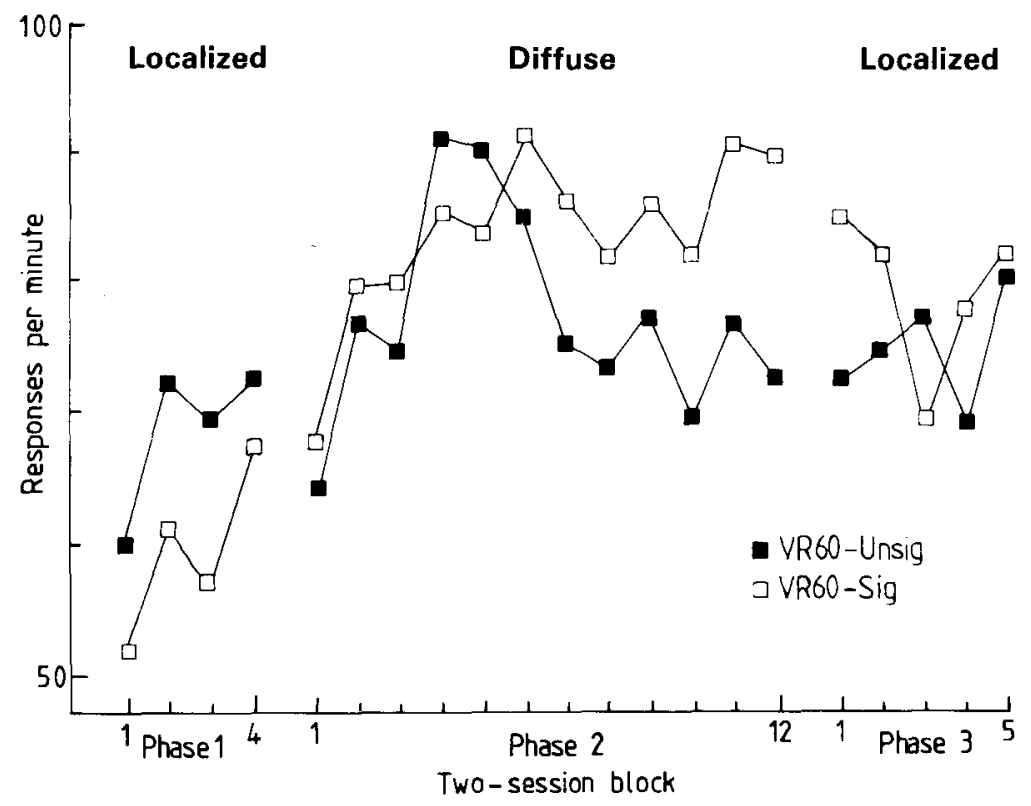

Figure 2. Results from Experiment 2. Mean response rates for diffuse and localized light stimuli groups, represented as two-session blocks. Unsig = stimulus presented uncorrelated with reinforcement; $\mathrm{Sig}=$ signaled reinforcement. 
that the use of a localized visual stimulus abolishes this potentiation effect. Thus, there is nothing about a visual cue per se that prevents potentiation from occurring. Rather, it is the use of a cue likely to promote autoshaped competing behavior that seems to be crucial. It remains possible, however, that the difference is effectiveness between the diffuse and localized cues reflects nothing more than a difference in salience between them. Experiment 3 was designed to investigate this possibility.

\section{EXPERIMENT 3}

In this experiment different groups of subjects were again trained with diffuse or localized visual stimuli as signals for reinforcement, as in Experiment 2. However, an attempt was made to assess the conditioned reinforcing properties that the stimuli acquired as a result of being used as signals on the VR schedules. In a final stage of training, food presentations were discontinued so that the level of responding maintained by the (response-contingent) light alone could be determined. If the localized light is ineffective in producing potentiation because it is only weakly associated with the reinforcer in animals that received this light as a signal, then response rates of these subjects should differ little from the rates of animals trained with uncorrelated presentations of the light.

\section{Method}

Subjects and Apparatus. Thirty-two male Lister hooded rats served as subjects. All were 6-8 months old at the start of the experiment; their ad-lib weights ranged from 275 to $360 \mathrm{~g}$. The subjects had previously served in an instrumental study investigating schedule dynamics, but had no previous experience of the stimuli and schedules used in the present experiment. The animals were housed in pairs, and were maintained at $80 \%$ of their free-feeding body weights. Water was available constantly in the home cage. The apparatus employed was that described in Experiment 2.

Procedure. Because of their previous experience, the animals needed no magazine or leverpress training. All subjects were exposed to the VR pretraining schedules described in Experiment 1. Following the final session of VR-20, the subjects were divided into four groups $(n=8)$ that were matched for response rates on the last session of VR-20. Two of these groups were to receive the localized light as a stimulus, and the other two groups were to receive the diffuse light. Of the two groups in each condition, one was designated to receive a signal that filled the 500 -msec delay of reinforcement, and the other two groups to receive the same delay of reinforcement but with a response-contingent $500-\mathrm{msec}$ stimulus presented randomly with respect to reinforcement. The experiment lasted for 20 sessions, each consisting of 30 trials. All other details of the procedure were as described in Experiment 1.

Following the completion of this phase of training, the groups all received two extinction sessions, during which both food and the stimulus were omitted. The next day following the last extinction session, food was again withheld, but the 500 -msec light stimulus was presented after every leverpress response for all groups. Each session lasted for $20 \mathrm{~min}$.

\section{Results}

Mean response rates for the four groups, represented as two-session blocks, are displayed in Figure 3. On the last day of pretraining, the mean response rates for the groups that were to receive the diffuse light were 37.9 (to-be-signaled group) and 38.5 (to-be-unsignaled group) responses per minute. Equivalent scores for the groups to be trained with the localized light were 38.3 and 38.7 responses per minute. An ANOVA with signaling condition and stimulus type as factors revealed no significant effects $(F \mathrm{~s}<1)$.

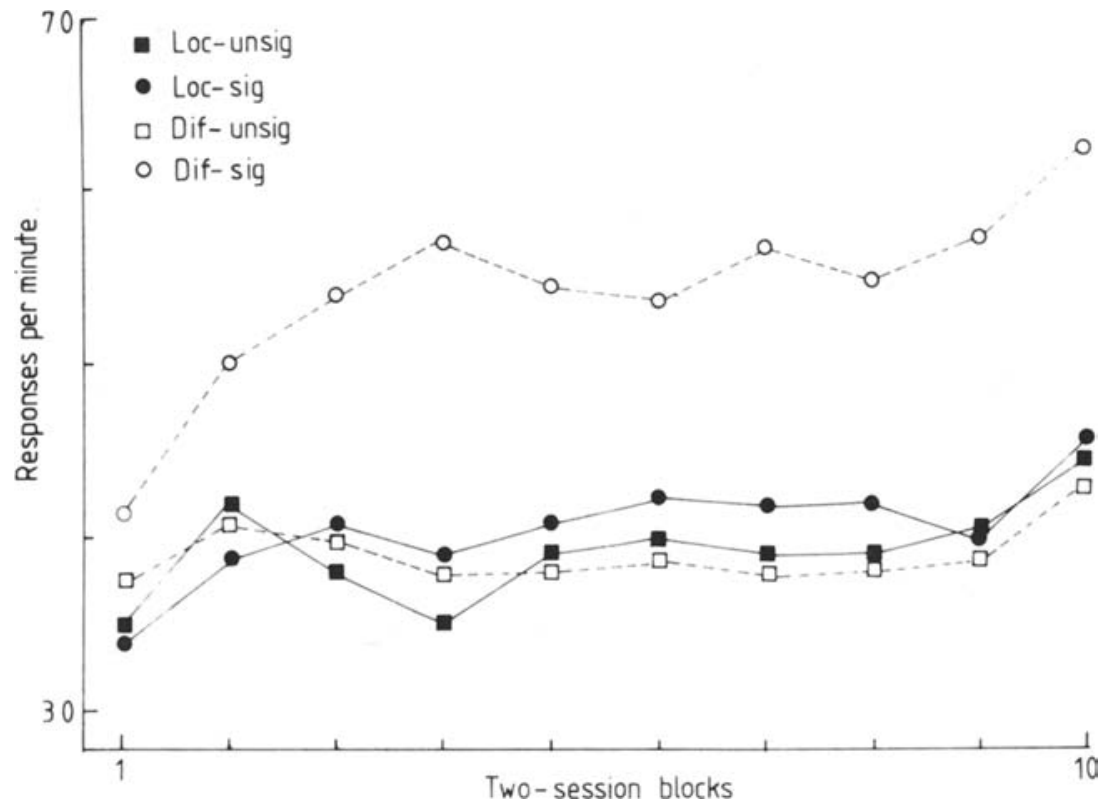

Figure 3. Results from Experiment 3. Mean response rates for the diffuse (Dif) and localized (Loc) groups receiving a signal correlated with reinforcement (sig), or a stimulus presented uncorrelated with reinforcement (unsig). 
Examination of the response rates displayed in Figure 3 for the two diffuse-light groups reveals that the subjects in the signaled reinforcement condition displayed a substantially higher rate of responding than did the subjects in the uncorrelated control group. However, this pattern of results was not evident in the localized-light groups. The subjects in the latter two groups responded at rates comparable to those shown by the uncorrelated diffuselight group. A three-factor ANOVA (signal $\times$ stimulus type $\times$ block) was conducted on the data represented in Figure 3. This analysis revealed no significant main effect of signal $[F(1,28)=1.57, p>.20]$ or of stimulus type $[F(1,28)=2.62, p>.10]$. The main effect of block, however, was significant $[F(9,252)=2.61, p<.01]$. None of the interactions between these main factors achieved significance $(p s>.20)$.

Inspection of the figure indicates a substantial numerical difference between the response rates generated by the diffuse reinforcement signal and those generated by its control condition. To confirm that the diffuse signal does come to support higher rates, separate analyses of the last three two-session blocks (two-factor, signal $\times$ block, ANOVAs) were conducted on the data from the diffuse- and localized-light groups. These analyses revealed, for the diffuse-light groups, a statistically significant main effect of signal $[F(1,14)=5.88, p<.05]$, but neither the main effect of block $[F(2,28)=2.18, p>$ .10] nor the interaction of these two factors $(F<1)$ proved to be significant. A similar two-factor ANOVA (signal $\times$ block) conducted on the two localized-stimuli groups, however, produced no significant main effect of signal or block, nor was the signal $\times$ block interaction significant $(p s>.20)$.

Mean response rates for the four groups on the second extinction session and on the test session are shown in Figure 4. Response rates in the extinction session were very similar for all groups. With the introduction of the light on the test session, however, a difference emerged between the groups that had previously received the stimulus correlated with food, and those that had received uncorrelated training. The latter groups showed a lowered response rate, reflecting the fact that the session constituted a further session of extinction. However, animals in the correlated groups showed an increase in responding, suggesting that the light was functioning as a conditioned reinforcer. This increase was evident in both correlated groups, and there was no sign of a difference between the diffuse and the localized light. This interpretation was supported by statistical analysis. A three-factor ANOVA (signal $\times$ stimulus type $\times$ session) was conducted on the data represented in Figure 4. This revealed a statistically significant main effect of signal $[F(1,28)=$ $11.60, p<.01]$ and a significant interaction between signal and session $[F(1,28)=18.04, p<.001]$. No other main effects or interactions proved to be statistically significant $(F \mathbf{s}<1)$. To investigate further the interaction between signal and session, the rates of responding on the extinction and test sessions were separately analyzed by two-factor ANOVAs (signal $\times$ modality). The ANOVA conducted on the response rates from the extinction session confirmed what is apparent from inspection of Figure 4; that is, there was no main effect on

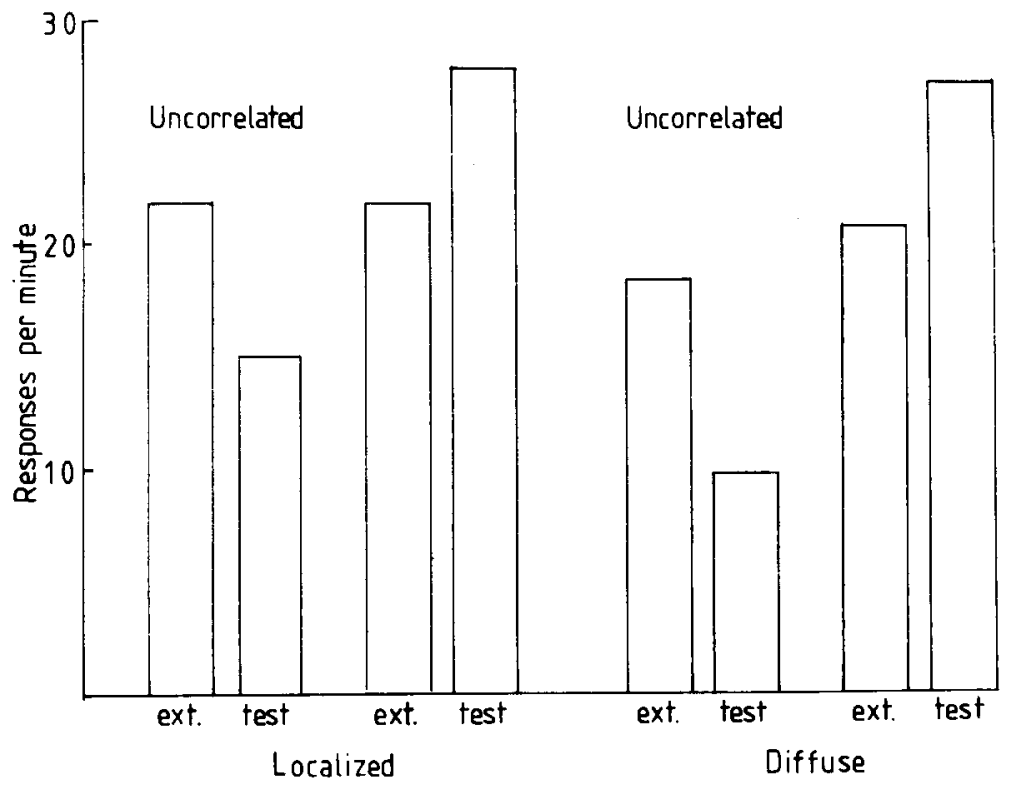

Figure 4. Results from Experiment 3. Mean response rates for both the correlated and uncorrelated groups that received the diffuse and localized light stimuli over the final extinction (ext.) and test sessions. 
response rates in extinction due to the previous signaling treatment or modality, and there was no interaction between these two factors $(F<1)$. The analysis of the response rates during the test session, however, revealed a statistically significant main effect of signal $[F(1,28)=$ $27.87, p<.001]$. The main effect of modality was not significant $(F<1)$, nor was there an interaction between these two factors $(p>.20)$.

These patterns of results lend no support for the suggestion that the localized light fails to produce the potentiation effect because it fails to form an association with the reinforcer. However, the possibility that the potentiation effect is obscured by the competing responses directed at the source of the light remains viable.

\section{GENERAL DISCUSSION}

The results reported here demonstrate that signaling reinforcement on a VR schedule by auditory or diffuse visual stimuli elevates response rates over those shown by groups receiving presentations of these stimuli uncorrelated with reinforcement. The potentiation effect reported by Reed et al. (in press) is thus confirmed. The present study also shows that potentiation is not evident in response rates when a localized visual stimulus is used as the signal, confirming the suggestion that the use of such a signal by Tarpy et al. (1983) played a role in their failure to find the effect. The localized light appears to acquire conditioned reinforcing properties as readily as does the diffuse light. This difference between diffuse and localized visual stimuli is consistent with the suggestion that competing responses (assumed to be more likely with a localized stimulus) are capable of obscuring the potentiating effects of the diffuse visual and auditory stimuli.

These findings have implications for the interpretation of the overshadowing effect produced by signaling reinforcement on a VI schedule. The majority of the experiments cited in the introduction to this paper used a localized visual stimulus in demonstrating the overshadowing effect. It has been argued that using such a stimulus as a signal for reinforcement evokes responses that interfere with leverpressing on the VR schedule, and there is no reason to suppose that such competition would not also occur with the VI schedule. However, it is not clear that interference from autoshaped responses is the sole explanation for the lowered response rate seen in the signaled VI procedure.

First, Roberts et al. (1985) made direct observations of the sign tracking evoked by a discrete signal associated with reinforcement on a VI schedule. They argued that even when the contribution from sign-tracking behavior is allowed for, the response rate of the animals receiving the signaled reinforcement remains lower than that of the subjects in the unsignaled condition. Second, it has been argued (and the experiments reported above produced results consistent with this argument) that the diffuse stimuli are less likely to produce interfering sign-tracking behavior than are localized stimuli. Nonetheless, the over- shadowing effect has been found when a diffuse auditory cue has been used as a signal (Pearce \& Hall, 1978, Experiment 4; Reed et al., in press, Experiment 3), and the size of the effect seems very little different from that produced with a discrete light as the signal.

Three possible explanations for the overshadowing effect were advanced in the introduction to this paper. The account based on sign tracking seems able to supply only a partial explanation. The original efficiency theory (see Roberts et al., 1984) was based on the apparent ineffectiveness of signaling reinforcement with a VR schedule, and is thus undermined by the results reported here (see also Reed et al., in press). A modified version of efficiency theory may, however, apply to the VR case. The enhanced rates of responding observed on ratio schedules with signaled reinforcement would lead to a greater number of rewards earned in a shorter period of time. If efficiency were expressed in terms of reinforcers per minute, then such a signal-induced potentiation of responding could be classed as more efficient. However, signaling reinforcement on a VI schedule did not enhance the number of reinforcers gained per minute in the study reported by Schachtman, Reed, \& Hall (1987, Experiment 1). Consequently, such a modification to the efficiency theory would need to state precisely what function of behavior is made more efficient by the signal, and to demonstrate that this function was enhanced in all cases. There remains the suggestion that the signal has its effect by detracting from the strength of a response-reinforcer association. The problem for this theory is that it supplies no grounds for predicting that the effect of the signal should be reversed when a VR schedule rather than a VI schedule is used. Some new account seems necessary.

One possibility worth brief discussion makes use of the notion that reinforcement acts upon "response units" rather than individual leverpresses. On a VR schedule the unit that typically precedes food delivery consists of a burst of responses, whereas on a VI schedule reinforcement often follows a single leverpress preceded by a pause from responding (Ferster \& Skinner, 1957; Morse, 1966; Pear, 1985). If the combination of conditioned and primary reinforcement presented in the signaled condition of these experiments is especially effective in promoting the emission of the appropriate response unit, then both potentiation and overshadowing might be expected, depending upon the schedule in operation. Clearly this suggestion needs to be specified more precisely if an adequate account is to be developed. One immediate problem that any elaboration needs to deal with is the recent demonstration by Schachtman et al. (1987) that potentiation can sometimes be found with a VI schedule (in particular, when it is arranged for the delay between response and reinforcer to be relatively long). The explanation offered by Schachtman et al. (1987) cannot be applied readily to the present results. They suggested that the effect of a signal for reinforcement depends upon the strength of the response-reinforcer association and that overshadowing will be found when the association is 
strong, but potentiation will result if the response-reinforcer association is weak. However, if response rate is taken as an index of the strength of the response-reinforcer association, then it must be concluded that the VR schedule generates strong associations. VR schedules have been found to generate two to three times the response rate of VI schedules, even when reinforcement frequencies are matched (Peele, Casey, \& Silberberg, 1984). The arguments offered by Schachtman et al. (1987) thus predict overshadowing, rather than potentiation. It remains possible, however, that different mechanisms underlie the apparently similar effects in the two sets of experiments.

\section{REFERENCES}

Dickinson, A., Peters, R. C., \& Shecter, S. (1984). Overshadowing of responding on ratio and interval schedules by an independent predictor of reinforcement. Behavioral Processes, 9, 421-429.

Edgar, D., Hall, G., \& PEARCE, J. M. (1981). Enhancement of foodrewarded instrumental responding by an appetitive conditioned stimulus. Quarterly Journal of Experimental Psychology, 33B, 3-19.

FERSTER, C. B., \& SKINNER, B. F. (1957). Schedules of reinforcement. Englewood Cliffs, NJ: Prentice-Hall.

FOREE, D. D., \& LoLoRDo, V. M. (1975). Stimulus-reinforcer interactions in the pigeon: The role of electric shock and the avoidance contingency. Journal of Experimental Psychology: Animal Behavior Processes, 1, 39-46.

HARSH, J., BADIA, P., \& RYAN, K. (1984). Signal modality and choice between signalled and unsignalled food. Journal of the Experimental Analysis of Behavior, 42, 279-289.

HeARST, E., \& JENkINS, H. M. (1974). Sign-tracking: The stimulusreinforcer relation and directed action. Austin, TX: The Psychonomic Society.

Holland, P. C. (1977). Conditioned stimulus as a determinant of the form of the Pavlovian conditioned response. Journal of Experimental Psychology: Animal Behavior Processes, 3, 77-104.

IVERSEN, I. H. (1981). Response interactions with signalled delay of reinforcement. Behavior Analysis Letters, 1, 3-9.

Karpicke, J., Christoph, G., Petersen, G., \& Hearst, E. (1977). Signal location and positive versus negative conditioned suppression in the rat. Journal of Experimental Psychology: Animal Behavior Processes, 3, 105-118.

Morgan, M. J., Lea, S. E. G., \& Nicholas, D. (1976). Choice of extinction signalling stimuli by rats. Leaming \& Motivation, 7, 31-49.
MoRse, W. H. (1966). Intermittent reinforcement. In W. K. Honig (Ed.), Operant behavior: Areas of research and application (pp. 52-108). Englewood Cliffs, NJ: Prentice-Hall.

PEAR, J. J. (1985). Spatiotemporal patterns of behavior produced by variable-interval schedules of reinforcement. Joumal of the Experimental Analysis of Behavior, 44, 217-223.

Pearce, J. M., \& Hall, G. (1978). Overshadowing the instrumental conditioning of a lever press response by a more valid predictor of the reinforcer. Journal of Experimental Psychology: Animal Behavior Processes, 4, 356-367.

Peele, D. B., Casey, J, \& Silberberg, A. (1984). Primacy of interresponse time reinforcement in accounting for rates under variableratio and variable-interval schedules. Journal of Experimental Psychology: Animal Behavior Processes, 10, 149-167.

Reed, P., Schachtman, T. R., Hall, G. (in press). Overshadowing and potentiation of instrumental responding in rats as a function of the schedule of reinforcement. Learning \& Motivation.

RoBERTS, J. E., TARPY, R. M., CoONEY, N. (1985). The effects of signaled reward on sign-tracking and response rate. Animal Learning \& Behavior, 13, 13-17.

RoberTs, J. E., TARPY, R. M., \& LeA, S. E. G. (1984). Stimulusresponse overshadowing: Effects of signalling reward on instrumental responding as measured by response rate and resistance to change. Joumal of Experimental Psychology: Animal Behavior Processes, 10, 244-255.

Schachtman, T. R., Reed, P., \& Hall, G. (1987). Enhancement and attenuation of instrumental responding by signals for reinforcement on a VI schedule. Journal of Experimental Psychology: Animal Behavior Processes, 13, 271-279.

ST. Claire-Smith, R. (1979). The overshadowing of instrumental conditioning by a stimulus that predicts reinforcement better than the response. Animal Learning \& Behavior, 7, 224-228.

TARPY, R. M., LeA, S. E. G., Midgley, M. (1983). The role of response-reward correlation in stimulus response overshadowing. Quarterly Journal of Experimental Psychology, 35B, 53-65.

TARPY, R. M., RoberTs, J. E., LeA, S. E. G., \& MidGley, M. (1984). The stimulus-response overshadowing phenomenon with VI versus FI schedules of reinforcement. Animal Learning \& Behavior, 12, 50-54.

TARPy, R. M., St. Claire-Smith, R., \& Roberts, J. E. (1986). The effect of informational stimuli on instrumental response rate: Signalling reward versus signalling the availability of reward. Quarterly Journal of Experimental Psychology, 38B, 173-189.

(Manuscript received March 2, 1987; revision accepted for publication June 12, 1987.) 